\title{
Performance Analysis of Proposed Congestion Avoiding Protocol for IEEE 802.11s
}

\author{
Kishwer Abdul Khaliq ${ }^{1}$ \\ Department of Production Engineering, \\ IGS, Universität Bremen, \\ Germany
}

\author{
Amir Qayyum \\ ${ }^{1} \mathrm{CoReNeT}$, \\ Capital University of Science and \\ Technology (CUST), Islamabad, \\ Pakistan
}

\author{
Jürgen Pannek \\ Department of Production Engineering \\ Universität Bremen, BIBA Bremer Institut \\ für Produktion und Logistik GmbH, \\ Germany
}

\begin{abstract}
The wireless technology is one of the core components of mobile applications with mobility support at low deployment costs. Among these, Wireless Mesh Network (WMN) is one of the technologies that supports mobile users for un-disrupted, reliable data connectivity, provides high bandwidth even in areas, where access of such services is difficult. Additionally, it features capabilities like self-configuring, self-healing, and self-organizing. IEEE proposed a MAC standard for WMN enhancements named IEEE 802.11s for multi-hop networks. Within this standard, the mandatory routing protocol called Hybrid Wireless Mesh Protocol (HWMP) is proposed for efficient utilization of resources to achieve high bandwidth at MAC layer. To improve this protocol, a congestion avoiding protocol was proposed, which utilizes alternate paths just before the congestion state is reached. The proposed technique does not add any overhead, it utilizes congestion notification frame, which is already part of standard. This paper discusses simulation results of the proposed routing protocol against the existing HWMP protocol for packet delivery fraction, throughput and delay. The results indicate that the proposed technique significantly improves performance of IEEE 802.11s.
\end{abstract}

Keywords-Wireless Mesh Network; IEEE802.11s; Congestion Control; Congestion Avoidance; Routing Protocol; HWMP

\section{INTRODUCTION}

The wide variety of interesting applications including broadband home networking, collaborative networks, building automation system, enterprise networking are using wireless technologies. These technologies are popular because of their attractive offered advantages, e.g. support for mobility and easy deployment. These are also used in number of strategic and smart health-care applications [1], commercial applications [2], automation [3] and disaster management [4]. Apart from the information and specific applications, currently users have more interest in use of general purpose applications like infotainment, online video gaming and streaming applications [5]. With the use of multimedia and interactive sessions, applications require high bandwidth and internet connectivity without latency. A Multi-hop wireless network is the best choice to fulfill user's need because it has the advantages of both ethos multi-hop and broadband access. This multi-hop wireless network is named Wireless Mesh Network (WMN), and it offers ease of deployment in rural and hilly areas to provide broadband services. The most efficient applications supported by WMN are broadband wireless access, industrial and business applications, smart health care, transportation management systems, production, hospitality, warehouses and provisional venues [6]-[10]. WMN is a special type of adhoc network with self-healing, self-configuring and self-organizing capabilities, and is also used for deploying wide variety of applications like e-applications [11], public safety and crises management applications [12], building automation control [13], emergency and safety applications [14]-[16].

In WMN, the mesh nodes have capability of a relay station and these nodes can communicate directly without involvement of central entity. Many solutions have been provided by different organizations for WMN [17], where it has three types of nodes. These are mesh clients, mesh routers and gateways. Furthermore, this technology can operate in three modes, i.e. infrastructure, client, or Hybrid. The first mode provides backbone access to the conventional clients and integration with existing networks [18], the second works in adhoc mode and the third is combination of both. The two-tier IEEE 802.11s standard is proposed by IEEE for WMN [19], which includes backhaul and access tier [20].

In IEEE 802.11s [21], adhoc mode allows communication between nodes without any central entity (i.e. Access Point (AP)). WMN in Figure 1 , gives an overview of WMN architecture in hybrid mode, basically it is a combination of Independent Basic Service Set (IBSS) and Extended Service Set (ESS). There are mainly three types of nodes, i.e Mesh point (MP), Mesh Portal (MPP) and Mesh Access Point (MAP). MP acts as a router excluding AP functionality, and use wireless links for connectivity to other nodes. Hence, internal mesh LAN is not an ESS. MAP has combined functionality of AP and MP. It gives association to the station. MPP includes MP and gateway functionality. MPP is responsible for handling entry and exit of MSDUs from WMN to other connected network. IEEE 802.11s MAC is the enhancement in the existing IEEE 802.11 MAC, but also have some additional functionality like routing protocol. This routing protocol is handled at MAC layer whereas layer 3 routing protocols are needed at MPP for path selection, while communicating with other networks [17]. The protocol stacks of the IEEE 802.11s on each type of type is shown in Figure 2.

The ongoing research is focusing on the functions of 802.11s MAC, which includes QoS (performing priority Control), congestion control and admission control. In addition to it, the enhanced MAC also includes a functions for achieving spatial frequency reuse, and to avoid performance degradation due to hidden and expose nodes problems [22]. To control congestion in network, many researchers consider its queue 


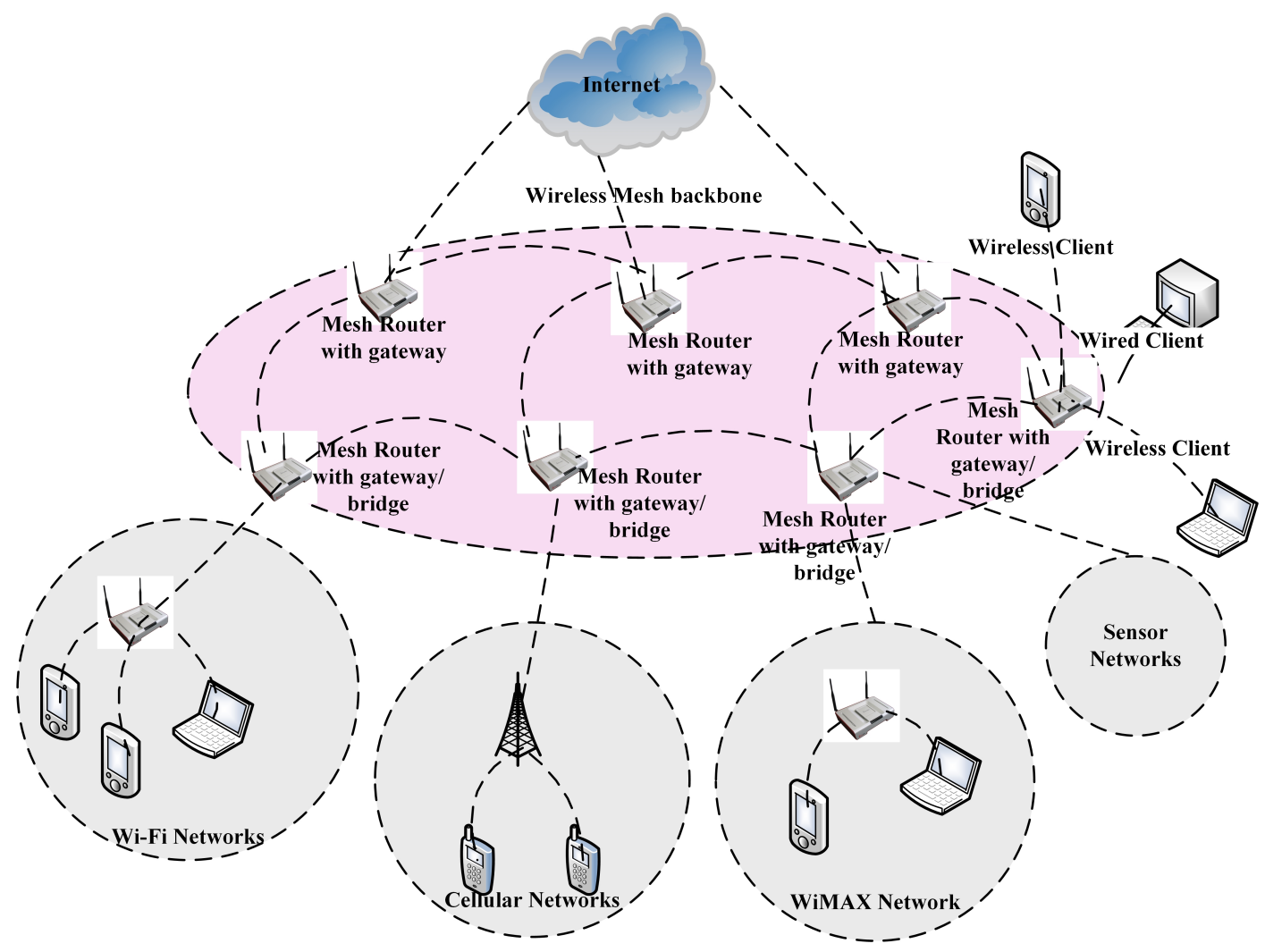

Fig. 1: Infrastructure/backbone WMN

mechanism, link capacity and routing protocols to get required results. Congestion is an important problem domain and it occurs when incoming network traffic load at a router is greater than the out going traffic rate [23]. In wireless scenario, it is predicted that packet lost or queue overflow may one of the reason for congestion. The reason behind this are the wireless communication issues which includes greater error rate due to wireless channel, wireless bandwidth etc. and the shared characteristics of the wireless channel. When packets are delayed due to mentioned issues, the increase in buffered packets in queue leads to congestion. In this regard, IEEE 802.11 s does not specified any congestion control mechanism. In literature, researcher proposed a number of congestion control mechanisms to resolve this problem, but every protocol has own limitations. In [24], we proposed a routing protocol to avoid congestion mechanism to address this problem which increase the throughput of the network. In this paper, we evaluate the proposed technique for congested scenarios and found that this protocol helps to reduce delay and packet loss. The remaining organization of the paper is as follows; Section II includes literature survey in details for congestion control protocols, Section III includes the proposed technique and Section IV includes the simulation and result analysis. Last Section $\mathrm{V}$ concludes our research work and discusses future work.

\section{Literature REVIEW}

All types of networks are facing Congestion issue, when they have to handle data more than the available capacity. It occurs when the incoming number of packets and outgoing number of packets are greater than the available network capacity. Network capacity is measured in term of network resources, available bandwidth or buffer of the network [26]. In wireless network, when one node has data to send, it accesses wireless channel and in this mechanism one node can transmit data at one time to share characteristics of it. Sometime, the increase in wait time to access wireless channel also increase packet delay and resulting queue length leads to congestion. As it is known that traffic is aggregated at the portal nodes, therefore, in a case of greater traffic load, MPs on the outer edges of the network suffer low throughput and greater packet loss in absence a congestion control mechanism [23]. An optional congestion control mechanism names hop-by-hop congestion control mechanism is outlined in the standard draft [21]. Each MP in hop-to-hop monitors congestion level by monitoring the incoming and outgoing traffic in its buffer. When the traffic load reaches at congestion specified threshold, the congested node should notify to its neighboring nodes to control their traffic. This mechanism includes three basic steps. The first step involves monitoring processes, in which each MP in network has to monitor its queue level for congestion rate or minimize the queue size by regulating the data traffic rate. The second step involves the notification on detection of congestion. The congested node broadcast Congestion Control Notification Frame (CCNF) to immediate nodes on its detection. The third step involves a control process. The nodes who receive CCNF, they limits their traffic rate according to service differentiation criteria. In addition to it, the CCNF also contains expiry information for 


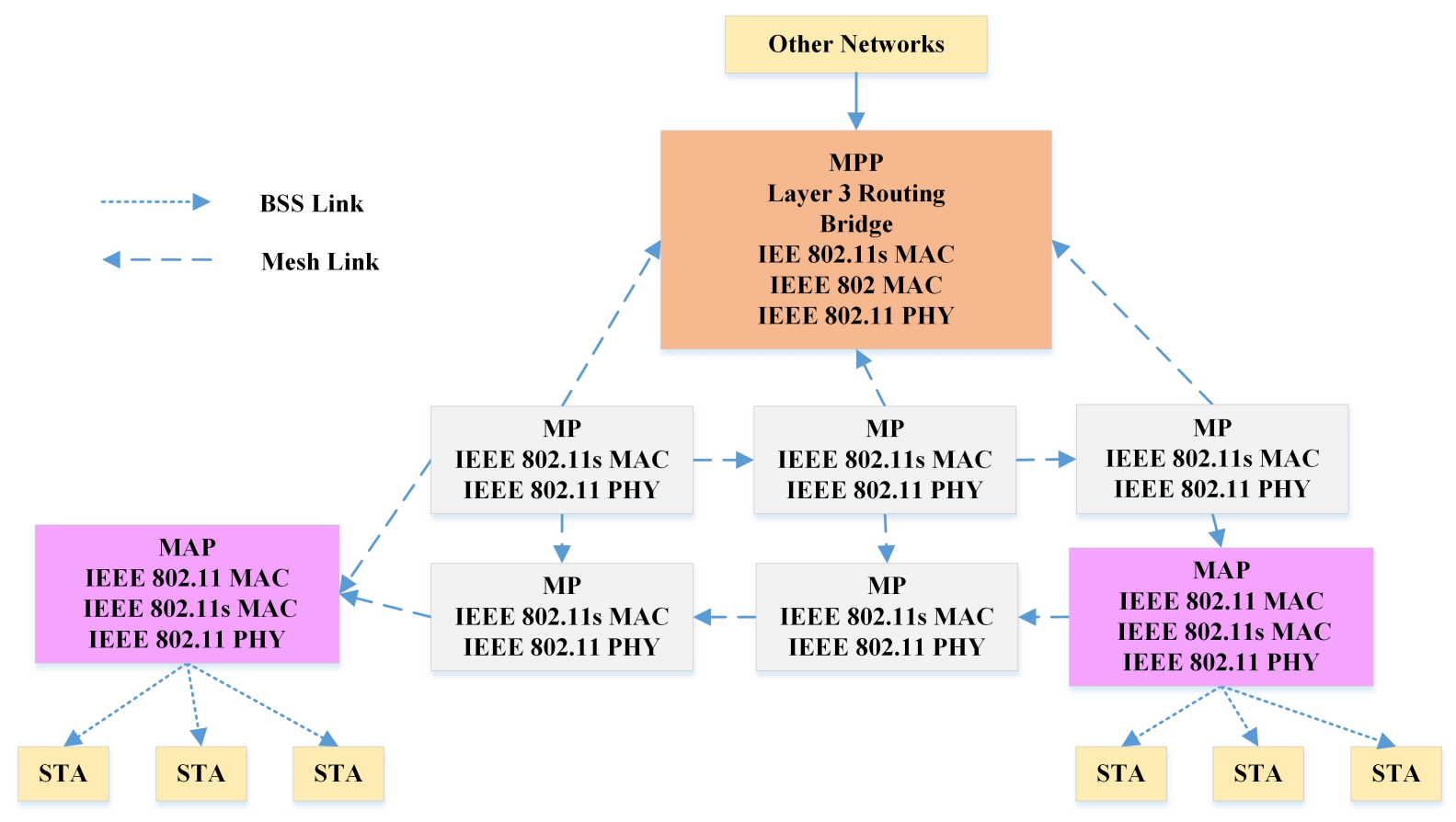

Fig. 2: Protocol Stack of IEEE 802.11s 25]

notification. In some cases, channel rate is also considered to restrict data-rate. Hence, a mesh node can also use this criteria for controlling data-rate [27].

In [28], authors proposed a modification in hop-by-hop mechanism by including feedback mechanism in distributed manner. This technique requires two NICs on each node at the same time, which operates independently. The mechanism is first derived algorithm for end-to-end, then it is further derived for hop-by-hop congestion control to control source rate endto-end. This algorithm works with the assumption of total knowledge of each flow on on each intermediate node. The controlling algorithm is responsible for monitoring incoming and outgoing transmissions and it performs computation on each relay node to sum all congestion states and maximum transmission rate for each flow. The intelligent part of it is that it selects smaller value for maximum possible rate for transmission. The drawback of this mechanism is additional cost for extra NICs and increase in overhead due to continuous feed back mechanism. There is an additional processing and synchronization cost because of combined algorithms to control congestion on each node.

In [29], the authors proposed another algorithm to provide end-to-end max-min-fairness to each flow. This co-ordinated congestion control algorithm is designed to deal with inters and intra-flows using multi-hop wireless links. On each wireless link, max-min-fairshare is computed continuously on assigned bandwidth and each flow uses allocated share in a fair way. In the whole mechanism a gateway is a central coordinator, which is used for traffic engineering. Similar type of mechanism is proposed in [30]. These both algorithms solve the issue of unfair channel sharing. However, these algorithms do not help in congested scenarios, because mechanism does not provide any feedback mechanism to limit traffic. In [31], the authors proposed a source based congestion control algorithm called WCP for multi-hop WMN, where source node is maintaining transmission information for each flow. It uses Additive increase and multiplicative decrease (AIMD) for controlling transmission rate. The algorithm uses WCPCap to estimate capacity of its neighbors and share among contented nodes. But the problem with this algorithm is that it is not providing any solution if a node receives and forward data for multiple nodes. Because of relay functionality and multiple flow maintenance on each node, the delay increases. Moreover, battery conceptions is greater due to additional computation for each flow on each node as compared to simple mechanism.

In WMN, there are two common type of congestions i.e. intra and inter-mesh congestion. Multiple algorithms are designed to resolve intra-mesh congestion. These algorithm also use congestion notification to control it. In [26] two algorithms i.e. Total Congestion Control (TCC) and Link Selective Congestion Control (LSCC) are proposed for intramesh congestion, but these algorithm do not provide very efficient solution. In TCC, CCNF is sent in local vicinity when congestion state reaches. The immediate nodes on receiving notification, block all traffic. In LSCC algorithm, on receiving congestion notification, the immediate nodes limit the traffic for specific link by blocking the data packets for a specific destination. An expiry time period is also included in CCNF, and flow resumed on expiry of notification. CCNF also contained information about the congested link, and when a node receives this frame, it blocks traffic only for the mentioned link. In [32], another algorithm is proposed which blocked traffic selectively. This algorithm is known as Path Selective Congestion Control (PSCC). This algorithm blocks traffic only for specified destination when a node receives notification on congestion occurrence. The CCNF frame includes information about specified flow. For the announcement of specific des- 
tination, this algorithm requires modification in the standard CCNF. Furthermore, on receiving modified CCNF, a node only blocks sending data for a specific destination, but it continues receiving for specified node. The scenario becomes more complicated when CCNF frame is further broadcast to immediate nodes in a continuous chain. These algorithms resolve congestion problem in few scenarios of multi-hop WMN.

Consider Figure 3 (a) for a congested scenario, it shows a congested link between mesh node $C$ and mesh node $D$. A queue size is monitored at node $C$, and when it is reached at the specified value, the node broadcasts the CCNF to the immediate nodes to limit traffic for node $C$. In the current scenario, node $E$ and node $B$ are in the neighbors. When these node receive notification, they stop transmitting data for node $B$ until the expiry of notification. In the mention scenario when we apply TCC algorithm, the immediate neighboring nodes stop data transmission but they continue reception from own neighbors. These nodes buffered all received data instead of forwarding till expiry of notification. If congested link could not resume from congestion, followed by another notification, then this delay cause congestion on neighboring nodes because of queued data. When these nodes also reach to a congestion state, and they also broadcast CCNF. If process continues, the whole network becomes congested.

In the same scenario when we apply LSCC, the immediate neighboring nodes stop data transmission for node $C$ until the CCNF time expired on receiving notification. During the notification expiry time, node $B$ queues all the received packets for the node $C$ only and forwards rest of the data traffic to other nodes. In this special case node $C$ also continues its local and global traffic transmission to its neighbors, which are node $E$ and node $B$ in this scenario. When the buffered data in the queue of the node $B$ reaches to the specified threshold, the node $B$ also broadcasts CCNF to its neighbors in vicinity. In the considered scenario, there neighbors are node $A$, node $F$ and node $C$. These nodes on receiving notification, block the traffic for node $B$. But the node $C$ whose traffic is already blocked due to last notification, will not receive this notification. In absence of any notification, the node $C$ as depicted in Figure 3 (b) continues its local data transmission or if already queued global traffic to node $B$, which results the packet lost. This situation becomes worst if node $A$ also becomes congested and broadcasts CCNF. As MPP is responsible for in-going and outgoing data in WMN, therefore MPs and MPP have the most congestion chances, specially for the case of bandwidth hungry applications. When it is occurred, packets overflow from buffer regardless of the number of hops the packets already have been traversed.

In this problem domain, authors in [24] proposed a technique called Congestion Avoidance Hybrid Wireless Mesh Protocol (CA-HWMP), which gives preference to avoids the congestion before its occurrence to improve packet delivery and improve network throughput. In this paper, we included the details about the proposed idea, algorithm and its limitations in Section III] Then we performed number of simulations to evaluate the behavior of CA-HWMP for different application data-rate for network throughput, packet delivery fraction and most important the end-to-end delay dealing with congestion issue. Section IV discusses all simulation results. Section D concludes all works with future directions.

\section{Proposed Mechanism}

Congestion Control mechanism works when congestion is already introduced in the network. The proposed mechanism focused on prevention of it. In WMN, routing is performed at the Data Link layer, and the proposed mechanism utilizes this routing protocol for congestion avoidance with small modification in basic mechanism. That is why it is named as Congestion Avoidance Hybrid Wireless Mesh protocol (CAHWMP) [24] and it uses HWMP in IEEE 802.11s with modification in mechanism for congestion avoidance. It includes three steps, monitoring, notification and intelligent re-routing. In first step, it monitors queue length on each node for each flow. The second step is to notify neighbors when it reaches to specified level. In the thirs step, the neighboring node calculate alternate path for destination by consider queue level to avoid congestion again. The proposed mechanism did not change the basic four information elements i.e. Path Request (PREQ), Path Reply (PREP), Path Error (PERR), and Root Announcement (RANN).

Consider a scenario depicted in Figure 4, it includes nine nodes in total. This scenario includes $G$ as a source node and $C$ as a destination node. Furthermore, node $A, F$ and $C$ are in the neighbor of node $B$. Node $G$ and $H$ are in the neighborhood of $A$. Node $G$ sends data to node $C$, the optimal path selected by its routing protocol is $G-->A-->B-->C$. In the selected best path, the immediate link to $C$ for a source node $G$ is node $B$. For a link $B-i C$, node $B$ monitors its queue length, when this queue length at node $B$ reaches at specified threshold, it broadcasts the CCNF frame to its immediate neighbors. The notified nodes, who have flow for node $C$ or through node $C$ performs reactive mechanism to find path for the specified destination excluding congested link. In the given scenario, the immediate neighbors of node $A$ are $G, H, F$ and $B$, which receive PREQ. Node $C$ and Node $G$ discard PREQ request, because first is congested and second is itself a source node. The PREQ is forwarded until it reached to the destination node. The destination node reply for the path by sending unicast PREP to the source. The new route establish from source mesh node $G$ to destination node in the given scenario is $G-->A-->F-->I-->C$. This procedure resumes data transmission via new calculated alternative link. This mechanism is not only good for the congestion avoidance but also reduce load-balancing at specific link/node in the multi-hop WMN. The queued data packet in the absence of congestion avoiding protocol, will now forward to destination node using this new established path. This mechanism reduces the packet lost, which was taking place due to queue overflow. This protocol allows the data transmission on the alternate route instead to wait the positive signal of congestion to restart transmission on existing track.

The proposed technique CA-HWMP which is basically a modification in the default protocol of IEEE 802.11s i.e HWMP. The Algorithm 1 works in active mode. Whenever a node have data to transmit, first it establishes a path for destination. For path selection, it broadcasts the PREQ to its immediate node. The receiving nodes forward the PREQ according to basic rules of the default protocol and additional to that they will check their queue level. If it is below to the 

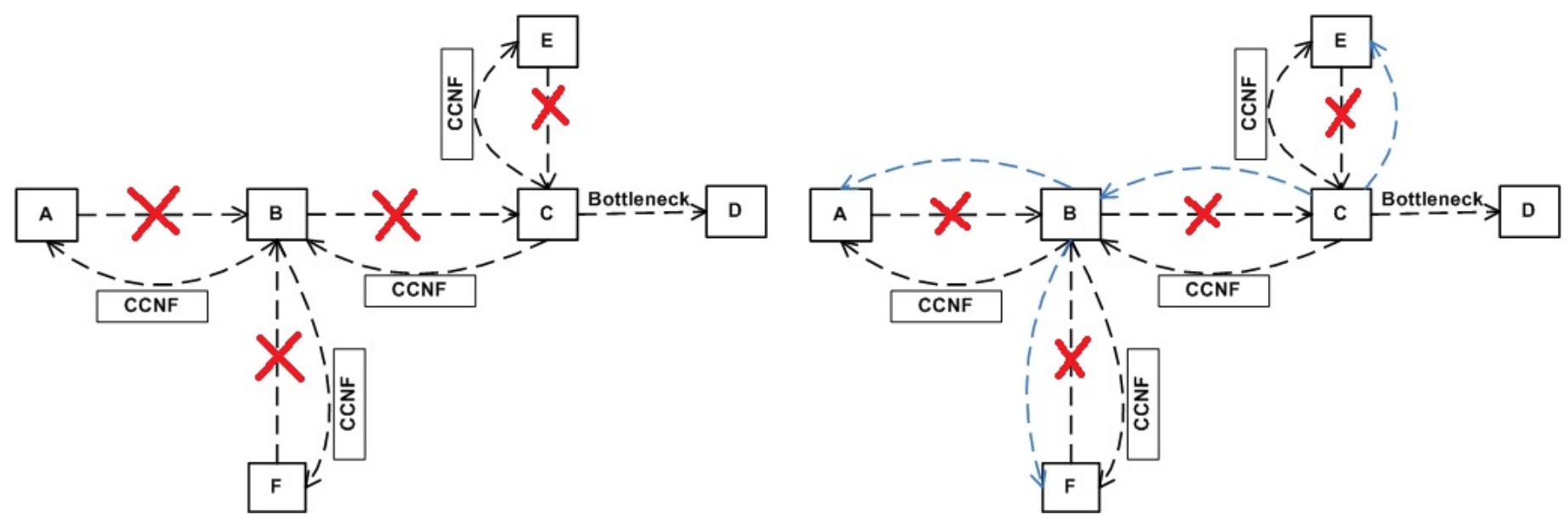

Fig. 3: (a) Congestion Scenario Using TCC; (b) Congestion Scenario Using LSCC;

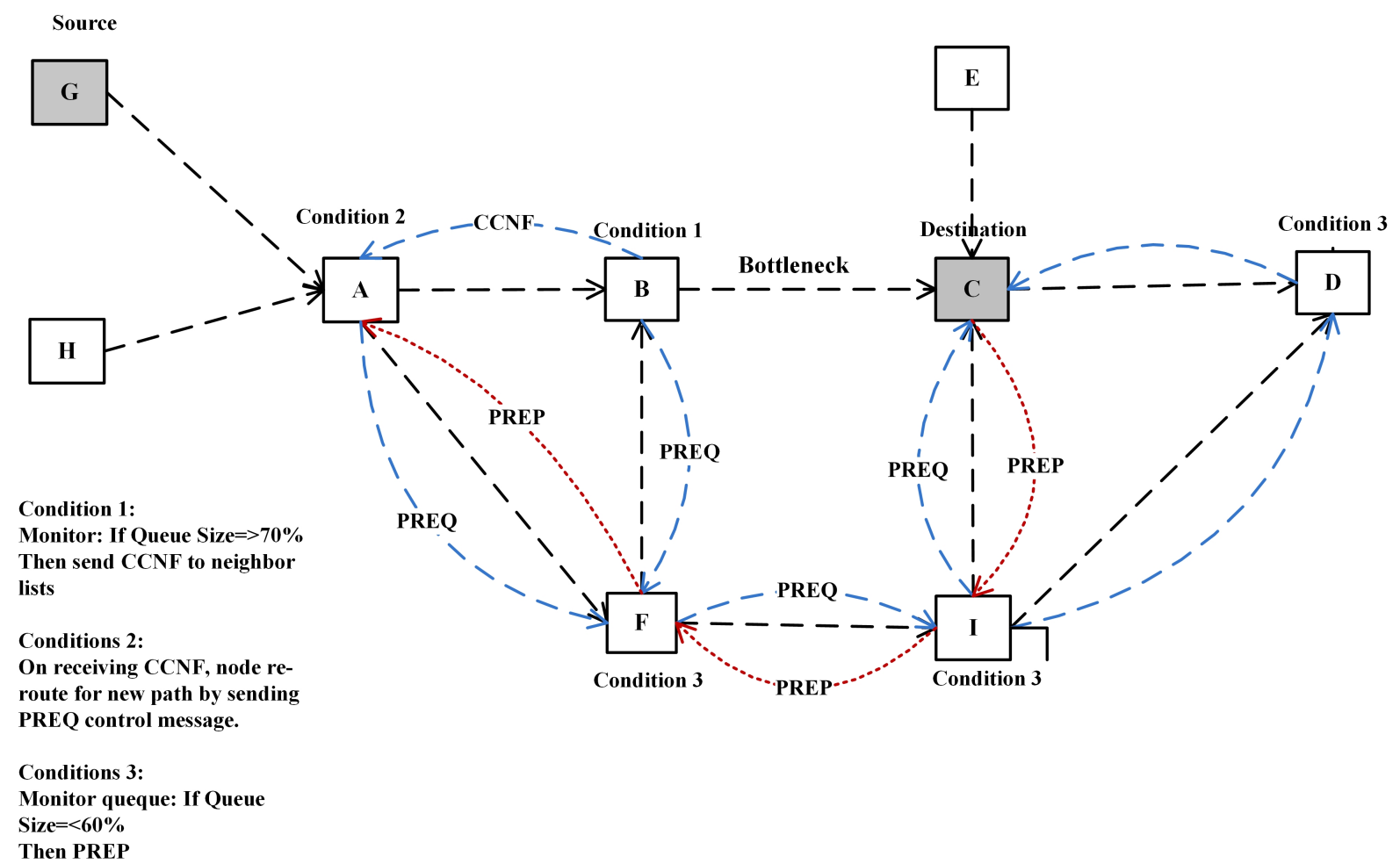

Fig. 4: CA-HWMP protocol Mechanism [24]

defined value, it forwards PREQ to other nodes. Finally, when the PREQ source node receives PREP, the path will establish.

Every mechanism has advantages and disadvantages. The proposed mechanism also has some limitations of CA-HWMP. Our proposed technique works well in a scenario, where we have possibility of alternate ways to re-route traffic. Although there are $80 \%$ chances of availability of alternate routes. Nonetheless, in the absence of alternate path, our proposed technique adapts standard available procedure. This protocol gives advantages of alternate routes. In absence of this technique, nodes received packets from neighboring nodes and queue them until CCNF expiry time reached. But in presence of it, utilization of alternate paths add benefits. It is doubted that the proposed protocol may have some scalability issue. It is the common practice, for route calculation few message exchanges between nodes in the network. If we have greater nodes in the network then this increase routing overhead. In a wireless network, with the increase of nodes in the network channel contention also increases and results in the increase in the wait time. 


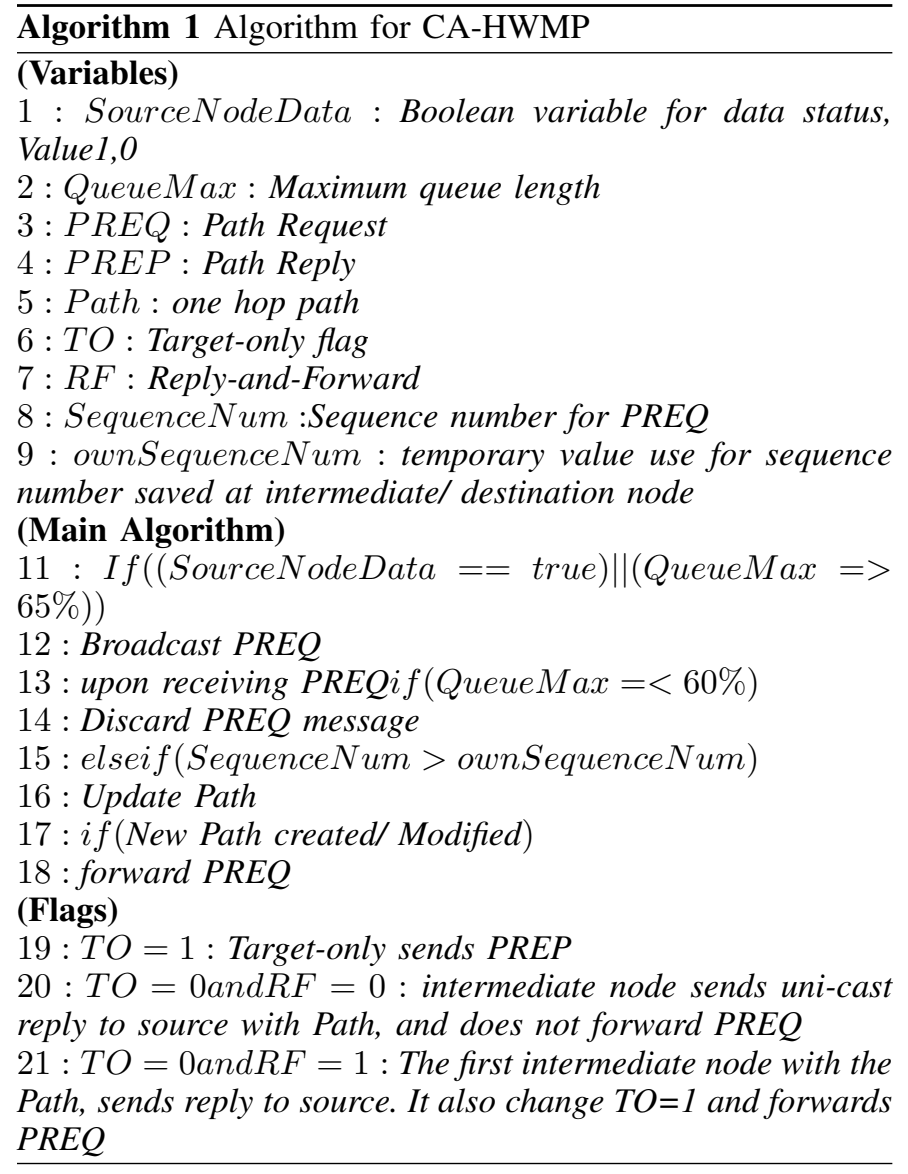

\section{Simulation And Result AnAlysis}

The main objective of this paper is to evaluate and discuss results of proposed CA-HWMP protocol in different scenarios. NS3 is used for protocol implementation, which is an open source simulator. It provides support and implementation flexibility of module implementation for wireless mesh network. By taking advantages of it, we patched CA-HWMP successfully into the already available mesh module using $\mathrm{C}++$, then we use scripts to evaluate protocol.

In Table I, the general simulation parameters are listed that we use in our selected scenarios. We perform simulation on the Linux Distribution Fedora Core using NS-3.14 version. It includes built-in supports of IEEE 802.11s and we used it as bench mark to compare with the implemented own module. To create congestion scenarios, we used On-off (CBR) application, which transmits data at a constant bit rate. During simulation scenario implementation, we focused on the queue level monitoring while increasing traffic rate slowly. Therefore, in our simulation scenarios, the used data-rate varies from $100 \mathrm{Kbps}$ to $350 \mathrm{Kbps}$ on UDP transport layer protocol. We started our simulator from 4 nodes and then we increases this number exponentially. Therefore we use grid topology for nodes positions. To observe closely, the number of nodes increases in both dimensions in each simulation scenario. The grid topology is represented in form of $\mathrm{X}$ and Y-axis as $m \times n$ where "m" represents the number of nodes on $X$-axis and " $n$ " in Y-axis. The distance between two nodes is $170 \mathrm{~m}$. Multiple simulation scenarios have been considered to observe the effect of application data-rate on throughput, packet delivery frac-
TABLE I: Considered Parameters for Simulation

\begin{tabular}{|l|l|}
\hline Operating System & Linux Distribution Fedora Core \\
\hline NS-3 version & NS-3.14 \\
\hline Wifi Standard & IEEE 802.11 s \\
\hline Mobility Model & Constant Position Mobility Model \\
\hline Number of Interface & 1 \\
\hline RTS/CTS & Disable \\
\hline Trace Module & Flow Monitor \\
\hline Traffic Flows & Constant-bit rate (CBR) \\
\hline Flows Varies (Kbps) & $100,150,200,250,300,350$ \\
\hline Packet Size (KB) & 1024 \\
\hline Transport Layer Protocol & UDP \\
\hline Routing Protocols at MAC & HWMP, CA-HWMP \\
\hline Number of Nodes in Grid & $4,9,16,25,36,49,64$ \\
\hline Transmission Range (m) & 170 \\
\hline Simulation Time (Sec) & 240 \\
\hline
\end{tabular}

tion (PDF), and end-to-end delay. The considered evaluating parameters are most effected in congestion scenarios. We have

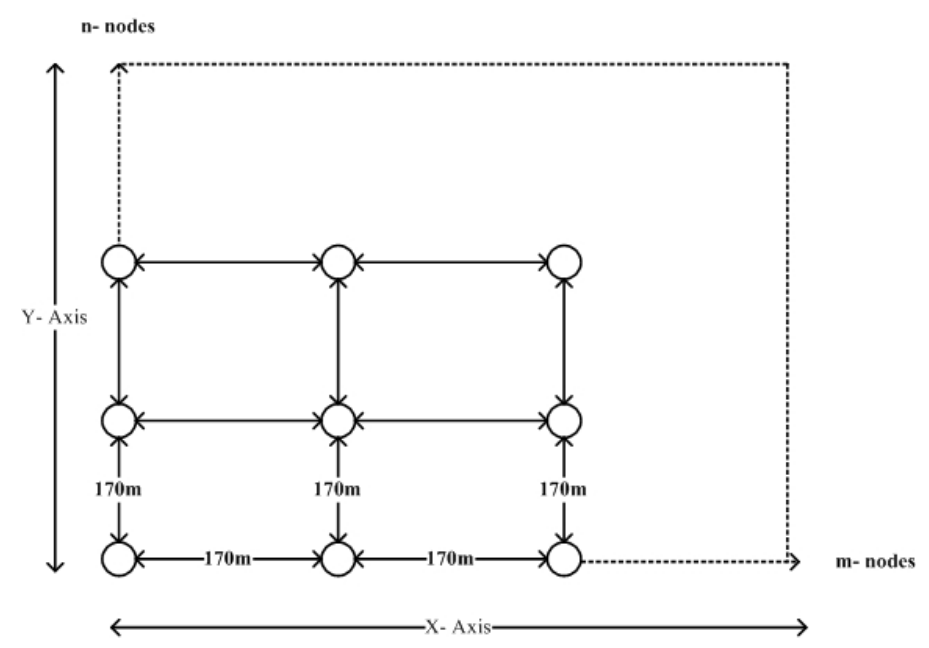

Fig. 5: $m \times n$ Grid Topology

selected multiple simulation scenarios, and in each scenario the possibility of multiple path is varied because the simulation is performed on varied number of nodes. The chosen grid topology $(m \times n)$ consisted of MP nodes. Figure 5 represents this topology where "m" indicates the number of the nodes in the $\mathrm{X}$-axis and $\mathrm{n}$ indicates the number of nodes in Y-axis. The first simulation run uses $2 \times 2$ grid then the increase in values of "m" and $n$ was additive.

\section{A. Effect of Application Data-rate on Throughput}

Throughput is one of the evaluating parameter in network simulations. To analyze the network behavior for this parameter, we fixed the traffic generating node. As the network contains different number of nodes along application data-rate variation, therefore we fixed the traffic generating nodes upto $50 \%$. To make scenario more realistic, we choose the source and destination nodes at run time. The nodes participate in path selection active and those node who have data to send use path selection. The reason behind to limit traffic flows up to $50 \%$ is that the utilization of alternative paths can be observed correctly. In this scenario, the used application is $\mathrm{CBR}$ where its traffic varies from $100 \mathrm{Kbps}$ to $350 \mathrm{Kbps}$, and the 
considered nodes varies from 4 to 64 . The maximum data-rate is $230 \mathrm{Kbps}$, therefore the device maximum data-rate is also fixed $350 \mathrm{Kbps}$. The computed value is the average throughput of the network. We generated graphs on computed value and graphs in Figure 6 (a) to (f) represent the network throughput on varied node density and data-rates. The variation in nodes in the grid topology is shown on $\mathrm{X}$-axis where grid varies from $2 \times 2$ to $8 \times 8$, while resultant throughput is shown on Y-axis.

The Graph in Figure 6 (a) shows network throughput for $100 \mathrm{Kbps}$ application data-rate, and the node density was from sparse mode to dense. Consider first case when the grid has 4 nodes, each node is in the direct access of one another and can transmit data directly with hop count zero. Then this grid size is increased from $2 \times 2$ to $3 \times 3$, the performance of both protocols were again similar. The reason is simple, in 9 nodes grid, node-1 sends data to node- 9 in the network; the intermediate node relays to the destination node. In this case, the best path has maximum one hop. The device has a capability to transmit is more than 3 times greater than the application data-rate. The only case is that, if more than 3 nodes transmit data using one relay node then the relay nodes queued the excessive packets. When this queue becomes full, it leads to congestion. We have very less nodes in the network, hence, are less chances of congestion on relay nodes. However, throughput of CA-HWMP negligible better than HWMP due to use of alternate path in a rare chance of congestion issue in this specific case. But when we increased mesh nodes from 9 to 16 , then the alternate path options were greater than the previous case. Here, the device maximum data-rate is same as in previous case. However, the relay intermediate nodes can be congested as they have higher degree of connectivity and multiple nodes can transmit data using single relay because of best path selection mechanism. In this case, when we have $100 \mathrm{Kbps}$ application data-rate, we observed little improvement because queued data remained below the threshold due to less application rate. This performance is changes when we increased mesh nodes in grid from 25, 36, 49 and 64. Due to increase in this number, there is also increase in data disseminating nodes, therefore, there is also an increase in network throughput. We observed that it increases while node density moves from sparse to dense mode. Both protocols graph slop shows the same trend, but the performance of CA-HWMP is better than the performance of HWMP as CAHWMP utilizes the option of second best path in case if best path is congested. Considering another scenario to observe network throughput with the increased value of application data-rate, which is increases from $100 \mathrm{Kbps}$ to $150 \mathrm{Kbps}$. The increment in number of nodes is same. The graph in Figure 6 (b) shows the throughput for this scenario. The simplest case is with 4 number of nodes, and transmission is simplest because all nodes are in the vicinity of one another. In the same scenario, the second case is with 9 mesh nodes and there is still less chances to use of alternate paths, and device rate is also 3 times greater than the application rate. Therefore throughput observed using both routing protocol is almost same. When mesh nodes are increased to 16, in case of both protocols, the availability of second best path also greater than previous case. As application data-rate is greater than the previous scenario, the relay nodes have still have greater margin of data forwarding in one time. The only possibility of packets drop from queue is, When queue becomes full. But if we continue to increase in number of relay nodes in grid, the graph shows the increasing throughput degradation behavior while using HWMP. The case when we have 36 nodes in a grid, with the increase in mesh nodes in grid, the availability of alternate path also increases. The best path also can have few hops to reach destination, and relay nodes may have to forward data on behalf of few neighbors. At relay node, the multiple flows can result in dropping packets from the queue. This packet drop ratio increases when more nodes enter in network to communicate. This degradation is even more in case of 49 nodes as compared to 36 nodes in grid, When we added more nodes in network, the new entering nodes generate more data to send/share in the network. However, by increasing mesh nodes in the network, there is also a increase in control overhead because of exchange of control messages. Furthermore, there is also an increase in contention of channel access, along the increase in frequency of data collisions and retransmissions. A relay node may drop packets due to queue overflow because of greater traffic load through them with the increase in node density. The graph in Figure 6 (b) presents two graph lines for CA-HWMP and HWMP, where CA-HWMP performance in term of throughput is better than HWMP. The reason is advantages of alternate paths at relay node, when an already selected path gets congested. In case of 64 nodes, this gain is maximum because of available alternate paths are also greater than previous cases which have less node density.

The above case discussed the $150 \mathrm{Kbps}$ application rate with the variation of node density. Now considering another graph in Figure 6 (c) with the increase in application datarate i.e. $200 \mathrm{Kbps}$. The simulation scenario continued with the increment of relay nodes as considered in previous scenario. Th graph lines shows the visible degradation of throughput with the use of HWMP in the whole scenario. First two cases, with 4 and 9 mesh nodes in network, the both protocols i.e. CA-HWMP and HWMP performs similar, But when the relay nodes vary from 16 to 49 , the degradation of throughput is visible because of increment in number of hops and relay traffic. In this case, where application has $200 \mathrm{Kbps}$ data-rate and a relay node can transmit with maximum $350 \mathrm{Kbps}$ datarate. The relay node has only capacity of transmitting data two nodes with current data-rate, and it queues the remaining data. Ultimately, the queue becomes full and drops packets from it. Results show that with the increase in nodes and intermediate hops, the throughput degraded due to overhead of control messages, collisions, and buffer overflow. Both protocol showed this trend, however, CA-HWMP shows the $13-18 \%$ gain in throughput depending upon network traffic.

In the next scenario, we increased data-rate from $200 \mathrm{Kbps}$ to $250 \mathrm{Kbps}$, and the graph in Figure 6(d) show the observed throughput with variation of node density. The first two case with 4 and 9 nodes are same as discussed in above scenario. In this scenario, an application sending data-rate is $250 \mathrm{Kbps}$ and node maximum sending data-rate is $350 \mathrm{Kbps}$. In case of 9 nodes, the one node is in the junction of all other nodes, which can be in the best part of each node involving one hop. When it receives data from multiple nodes, initially it queues data, then eventually drops it from the queue due to buffer overflow. It drops to network throughput. In case of CA-HWMP, when number of nodes varies from 16 to 64, its performance is better than HWMP due to alternate paths availability. The traffic is re-routed to alternate route, if the 

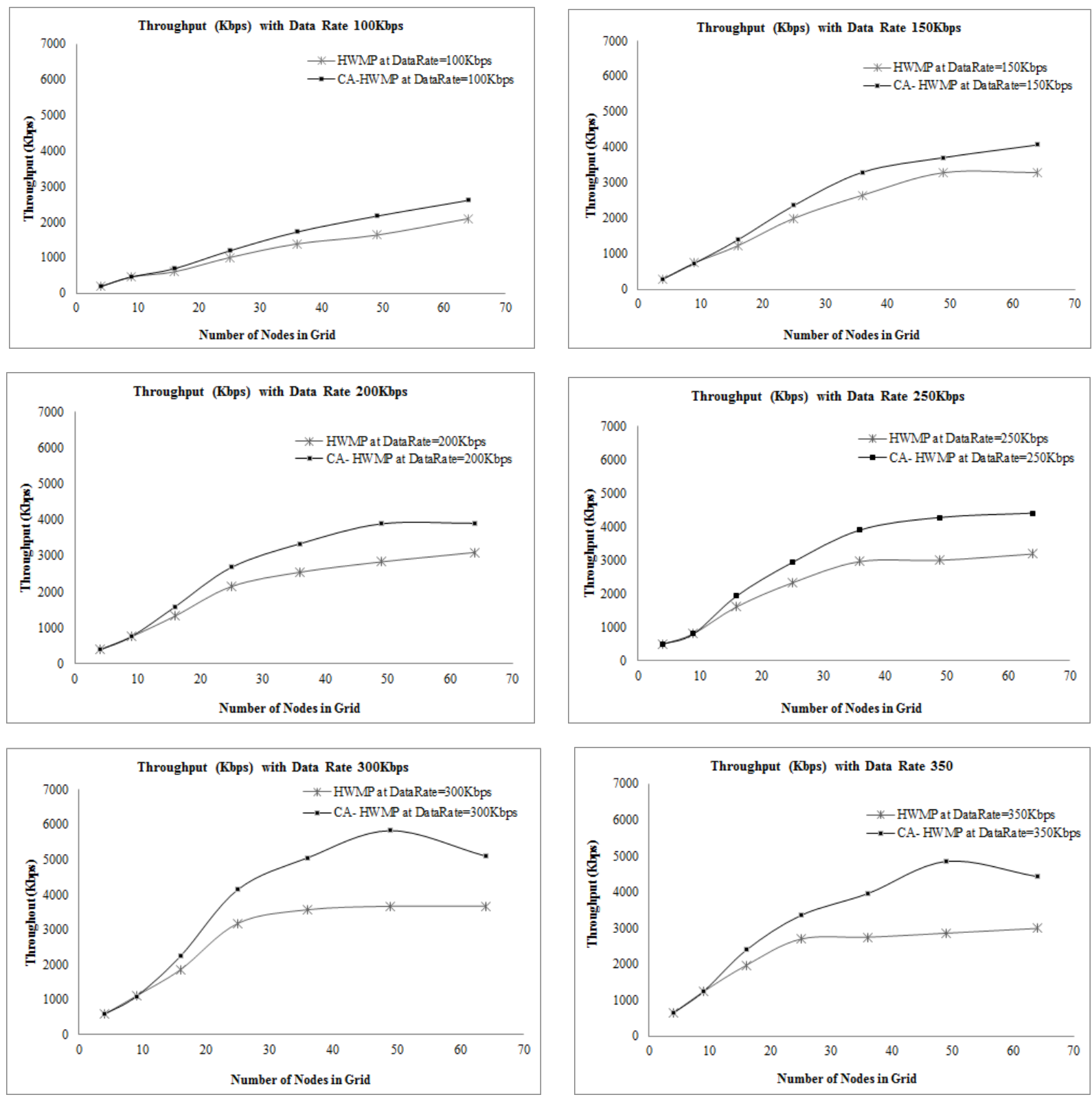

Fig. 6: Throughput Comparison of HWMP and CA-HWMP 300Kbps;(f) 3500Kbps

first best path is congested. CA-HWMP graph line shows that when we increase the number of nodes in a grid from 49 to 64, this improvement is almost equal to as we achieves in 49 node grid. Although, CA-HWMP gives possibility of traffic re-route, but when nodes in the network and generated traffic increase upto a level, the high control messages, collision and interference reduce the overall performance of the network. In any case, CA- HWMP is performing better than HWMP.

In Figure 6(e), the graph shows the trend of throughput in the network with $300 \mathrm{Kbps}$ application daterate. In the case of 4 nodes, the performance of both nodes is similar. But with the increase in the mesh nodes from 4 to 9 , throughput drops due to network topology and middle relay node selection for mostly best path. In this case, application data-rate is almost equal to the device data rate, therefore relay has the capability to transmit one node data at one time, and if it has to transmit more than one node data, the it maintained queues. This can lead to queue to overflow. As there are less possible paths in case of congestion, hence there is no real advantage of using CA-HWMP in this scenario. Both protocols perform almost likewise. The gain in throughput is visible, when we increase relay node nodes from 9 to 16 in case of CAHWMP. The gain in throughput is more visible, when further increase nodes i.e. $25,36,49$. The graphs show that with the increase from 36 to 49 , performance difference between both protocols is most significant. Considering HWMP in this scenario, the performance drops due to relay traffic at relay nodes. In the same scenario CA-HWMP performs good, 
because it shifted traffic on the alternate second best path. CAHWMP performance is on peak with 49 nodes in network. At the same time, the graph also shows the stable performance with 64, and the gain is less than the 49 node's grid. Although CA-HWMP exploits the possibility of alternate paths, but increase in the mesh nodes also increase control messages, channel contentions and re-transmissions.

In the last scenario, we fixed application data-rate exactly equal to the device data-rate to observe the behavior of network. First two scenario are same, as discussed in previous cases. In the third scenario, when we increase mesh nodes to 16 , both routing protocols perform good, but CA-HWMP performance is better than HWMP. When mesh nodes vary from 16 to 49, HWMP performance degraded. But CA-HWMP performs better than HWMP, due to the benefits of alternate paths, and re-route traffic on second optimal path when first gets congested. With 64 nodes in the network, throughput degrades in scenario of HWMP protocol because with the expansion of the network, the number of hops also increase between source and destination (Assuming first node is a source node and last node is the destination node). Therefore, the relay nodes may drop packets from queue as arrival rate is greater than the device forwarding data-rate. Though, CA-HWMP is performed better, but the gain in throughput is not good. Its performance is also affected by exchange of control messages, routing protocol messages and channel contention. The graph in Figure 6(f), shows that CA-HWMP is performance is better than HWMP, it also shows the decline in throughput when we increase the number of nodes in grid 49 to 64 , even in case of CA-HWMP. In case of 49 nodes grid, CA-HWMP performance is most significant.

We observed the network throughput by running simulation of number of scenarios. It is observed that the network throughput is increased with the increase an application datarate. We also observed that the performance of CA-HWMP is better than HWMP with the increase in application data-rate. But the increase in throughput is limited to specific number of hops and network traffic. We observed maximum throughput when we have $300 \mathrm{Kbps}$ application data-rate and 49 mesh nodes in the grid. All graphs show that the performance of CA-HWMP is better when the number of nodes in network are between 25 to 64 as compared to HWMP and the maximum gain is at $300 \mathrm{Kbps}$ data-rate in the network of 49 node grid. This shows that the performance is limited to the limited hop count for congestion avoidance.

\section{B. Effect of Application Data-rate on Packet Delivery Frac- tion $(P D F)$}

The second evaluation paramer, that we consider for evaluation of our proposed mechanism is Packet Delivery Fraction (PDF) to examine the network behavior on increasing datarate. This PDF is achieved by computing percentage receiving data-rate at receiving nodes. Figure 7 from (a) to (e) show the graphs of PDF for both considered protocols. In the graphs, $\mathrm{X}$-axis represents the number of nodes in the network, while Y-Axis represents percentage value of PDF. By using these values, we monitor the actual gain in proposed technique.

When the number of nodes increases in the grid, the PDF decreases due to increase of intermediate hops, contention for channel access and control overhead. In-spite these factors, with the increase in flows, the PDF decreases on relay nodes due to buffer overflow in absence of congestion control mechanism in HWMP.

Consider a scenario, where application has data-rate of $100 \mathrm{Kbps}$, device transmitting rate is $350 \mathrm{Kbps}$ and relay nodes vary from 4-64. The graph in Figure 7 (a) shows that both protocols has decreasing behavior on increasing number of mesh nodes. However, this trend is less in case of CAHWMP. First two cases are similar to the throughput case. The forwarding rate of device is more than three times of application data rate and mostly nodes are in direct access of each other, therefore this decrease is normal in both protocols. In case of $4 \times 4 \mathrm{Grid}$, this ratio drops due to increase in hop count between source and destination and also because of other factors like channel contentions, collisions and retransmissions. With the increase of mesh nodes in the network, this drop also increase. The best path selection mechanism in HWMP is hop count. In this scenario, nodes only select path with minimal hops and ignore the device transmitting capacity and queue length. Mostly traffic pass though the shortest path, in few scenario a single node becomes a bottleneck, which leads to packets drop. In CA-HWMP, nodes consider queue length during path selection and utilize alternate path as well, if congestion occurs during transmission. Results also show that CA-HWMP helps to improve gain in PDF. In the next scenario, the considered topology as used in previous scenarios. We kept all all other parameters constant and only changed application data-rate $150 \mathrm{Kbps}$. The graph lines in Figure 7(b), presents no significant difference observed between both protocols when we have 4 and 9 nodes grid. With the increase in mesh nodes from 9 to 16 along 50\% traffic flows, the CA-HWMP PDF gain is greater than HWMP due to the greater possibility of alternate paths as to $2 * 2 a n d 3 * 3$ grid. In case of 25 mesh nodes, though more nodes are available to disseminate data, however PDF gain in CA-HWMP is better as compared to HWMP. The simulation results show that $15 \%$ packets are dropped due to buffer overflow and $12 \%$ packet lost because of wireless reasons HWMP. With the use CA-HWMP this PDF increases 14\%. In a scenario with 36, 49 and 64 mesh nodes, decrease in PDF is greater than the previous scenarios. In case of 64 mesh nodes, this PDF degradation is observed $50 \%$. The reason is that packets drop from the queue due to greater difference between incoming and outgoing traffic rate. But the buffer overflow on relay nodes is not only reason, packets also drop due to wireless channel access, interference, packet collisions. However, the gain in PDF is improved in all scenarios as compared to HWMP.

The gain in the PDF shows that performance of mesh network is improved with the use CA-HWMP instead of HWMP. This improvement is achieved by utilizing alternate best paths when the best pat is blocked due to network congestion. But overall decline in PDF with the increase of network diameter is also because of channel contention, control messages overhead, and increase in number of hops. Consider another scenario and graph shown in Figure 7 (c) where the variation in network topology is same but the application datarate is changes to $200 \mathrm{Kbps}$. All other network parameters are kept constant including application data rate, and we observed the PDF but we changes the grid topology of 4 and 9 nodes, the PDF value in both routing protocols is almost same. In 

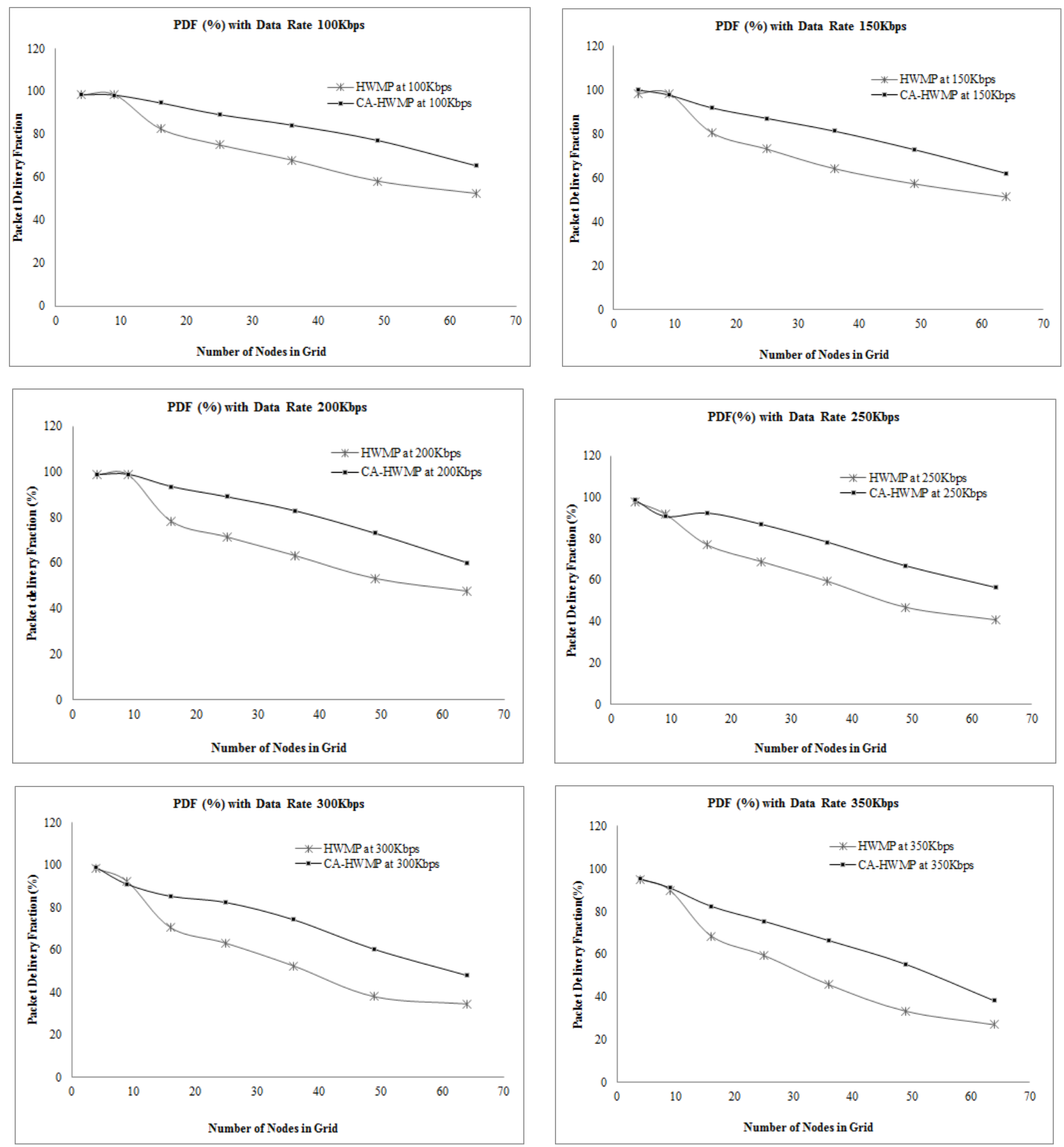

Fig. 7: PDF Comparison of HWMP and CA-HWMP at (a) 100Kbps; (b) 150Kbps;(c) 200Kbps;(d) 250Kbps;(e) 300Kbps;(f) $350 \mathrm{Kbps}$

case of HWMP routing protocol, by increasing mesh nodes to 16 , this ratio decreases to $76 \%$ due to increase hop count between sender and receiver. When relay nodes receive data from multiple nodes, if this receiving rate is greater than the transmitting rate, they queued received data, later this situation leads to congestion. However, decline in PDF is not because of congestion. With the increase in network nodes, the network traffic load also increases, control messages traffic and due to interference, the chances of collision also increases. All these factors lead together in PDF decline. When we have 16 nodes in the network, the statistics show that there is about $15-17 \%$ packet lost due to congestion while remaining packet lost is due to other factors. When relay nodes in the network varies from 25 to 64, this decline increases due to mentioned reasons.

In case of CA-HWMP in the same scenario, we observed significant improvement in PDF value. Although, PDF decreases with an increase in relay nodes, however this decline is less than HWMP. In these scenarios, the PDF gain increases 
due to possibility of alternate paths. However, graph in Figure 7 (c) shows that CA-HWMP performs better when mesh nodes varies from 16 to 64 , and it performs best when there are 49 node network with $50 \%$ traffic load.

Consider another scenario where we fixed application datarate $250 \mathrm{Kbps}$, but mesh nodes vary from 4 to 64 with $50 \%$ network traffic. First scenario with 4 nodes grid is always simple. In case of 9 mesh nodes, topology allows one in junction, can be a bottle-neck due to data forwarding. In this scenario, the device transmitting rate is $350 \mathrm{Kbps}$, while application rate is $250 \mathrm{Kbps}$ where relay nodes maintain queues to handle incoming data. At one point, these queues get congested and may result in packet loss. When we have 16 mesh nodes in the network, the PDF value decreases to $76 \%$, and which further increases by increasing mesh nodes. However, the simulation results show that CA-HWMP performs better than HWMP due to possibility of alternate paths, this difference is visible in Figure 7 (d).

The graph line of CA-HWMP in Figure 7 (d) shows the increase in PDF gain when new nodes enter into the network, but this gain decreases when we have 64 mesh nodes in this scenario. With the increase in mesh nodes in grid, there is also increase in node interference, channel contention, packet collisions and control overhead, which lead to the decline in PDF.

Consider a scenario with $300 \mathrm{Kbps}$ application data-rate and nodes vary from 4 to 64 . The performance of both protocols is good in the grid of 4 nodes. The decline in PDF is observer when we increase mesh nodes to 9 and further, where this value decreases due to multi-hop. The queue management issues, channel contention, interference on each relay nodes effects the PDF. The graph lines of CA-HWMP and HWMP in the same scenario show that the performance is similar, because in this scenario there is less possibility of alternate path in case of congestion. When we vary node density by adding new nodes in the network i.e. 16, 25, 36, 49 and 64 nodes, the decline in PDF is greater in both routing protocols. However, this decline in CA-HWMP is less than HWMP. The CA-HWMP performs only better, when there is availability of second best path when already existed becomes congested. Therefore, we observed this PDF gain with increases in mesh nodes.

In the graph shown in Figure 7 (e), X-axis represents nodes the network and Y-axis represents nodes PDF. The graph presents the decline in PDF with the increase in mesh nodes in the network while using both routing protocols. But this decline is greater in HWMP as compared to CA-HWMP. In this scenario, we set application data-rate $300 \mathrm{Kbps}$, and varied grid topology of 4 to 64 nodes. This scenario include application data-rate is almost equal to the device transmission rate i.e. $350 \mathrm{Kbps}$. In the first two scenarios, both protocols perform alike. The simulation results show that when we increase mesh nodes from 9 to $16,25,36,49,64$ in the grid, $\mathrm{PDF}$ decreases due to greater data-rate. The relay nodes receive data from multiple nodes, when their receiving rate becomes greater than the device transmission rate. Therefore, the queue becomes full and drops packet. In Figure 7(f), the graph lines of both protocols show the same behavior that we observed in the last scenario.

Through the simulation results of these multiple scenarios, we concluded that the PDF values depend on an application data-rate and maximum limit relay node to transmit data. If the application generates data-rate greater than the device maximum transmission-rate than packets drop at the application layer. If the application data-rate is less or equal to the device transmission-rate then no packet drop at application layer. In the evaluating scenarios, if there is only one hop involved between source and destination, then there is less probability of packet drops. With the increase in involved relay nodes, this ratio increases due to multiple factors which may include queue, channel contention, interference and control overhead. In our scenario, sending and receiving nodes are chosen randomly, MPs forward data for these nodes. When a MP forwards data on behalf of more than 2-3 nodes then the queue may reach to maximum level then the packets drop from it, resulting in decline of PDF in the network. However, CA-HWMP behaves different from HWMP. It monitors queue level, when it reaches specified threshold, it re-routes data on alternate path. Therefore, there is improvement in PDF while using CA-HWMP. The graphs shown Figure 7 are drawn for multiple scenarios with at different data-rates and they represent PDF difference while using CA-HWMP and HWMP.

\section{Effect of Application Data-rate on End-to-End Delay}

End-to-end delay is one of the important evaluation parameter. In multi-hop networks, where nodes relay data on behalf of neighbors, they maintained queues. Due to wireless medium, each relay node waits for channel access to transmit data, which adds delay in the packet deliver. To observe the delay in the network, we used different application data-rate with different node density. We computed end-to-ed delay (s) while varying data-rate from $100 \mathrm{Kbps}$ to $350 \mathrm{Kbps}$ and mesh nodes from 4 to 64 . The considered parameters are listed in Table I] We used grid topology with the dimension of $m \times n$ as presented in Figure 5. The "m" in the grid is number of mesh nodes on X-Axis and "n" on Y-Axis. In these scenario, we have $n=m=1,2,3,4,5,6,7,8$, hence, the grid varies from $1 \times 1$ to $8 \times 8$.

Consider a scenario with $100 \mathrm{Kbps}$ application data-rate and mesh nodes varies from 4 to 64 in the network. In teh first subscenario with 4 nodes of grid, and these are directly connect access to one another, therefore observed delay is negligible. When we increase nodes in the grid, the increase in delay is greater than previous scenario due to increase in hop count, channel contention, collisions and re-transmissions as depicted in Figure 8 (a). In the next scenario we increased data-rate to $150 \mathrm{Kbps}$, and the Figure 8 (b) presents end-to-end delay for HWMP and CA-HWMP protocols. The bar lines indicate that the delay increases with the increase in number nodes. Initially, when grid has less nodes in the network, the observed delay was also less, but with the increase in nodes, the observed delay is greater as compared to last scenario. The reason is that, with the increase in nodes in the network, the number of hops for packet traversing are also increases, and if a relay node is responsible for forwarding data on the behalf of multiple node, then due to maintained queue this delay also increased. If the device transmission rate is less than the receiving-rate then more delay added for transmission. The nodes queued packets, and forwarded when they have channel access to transmit it. The queued data increases delay, and if multiple hops are 

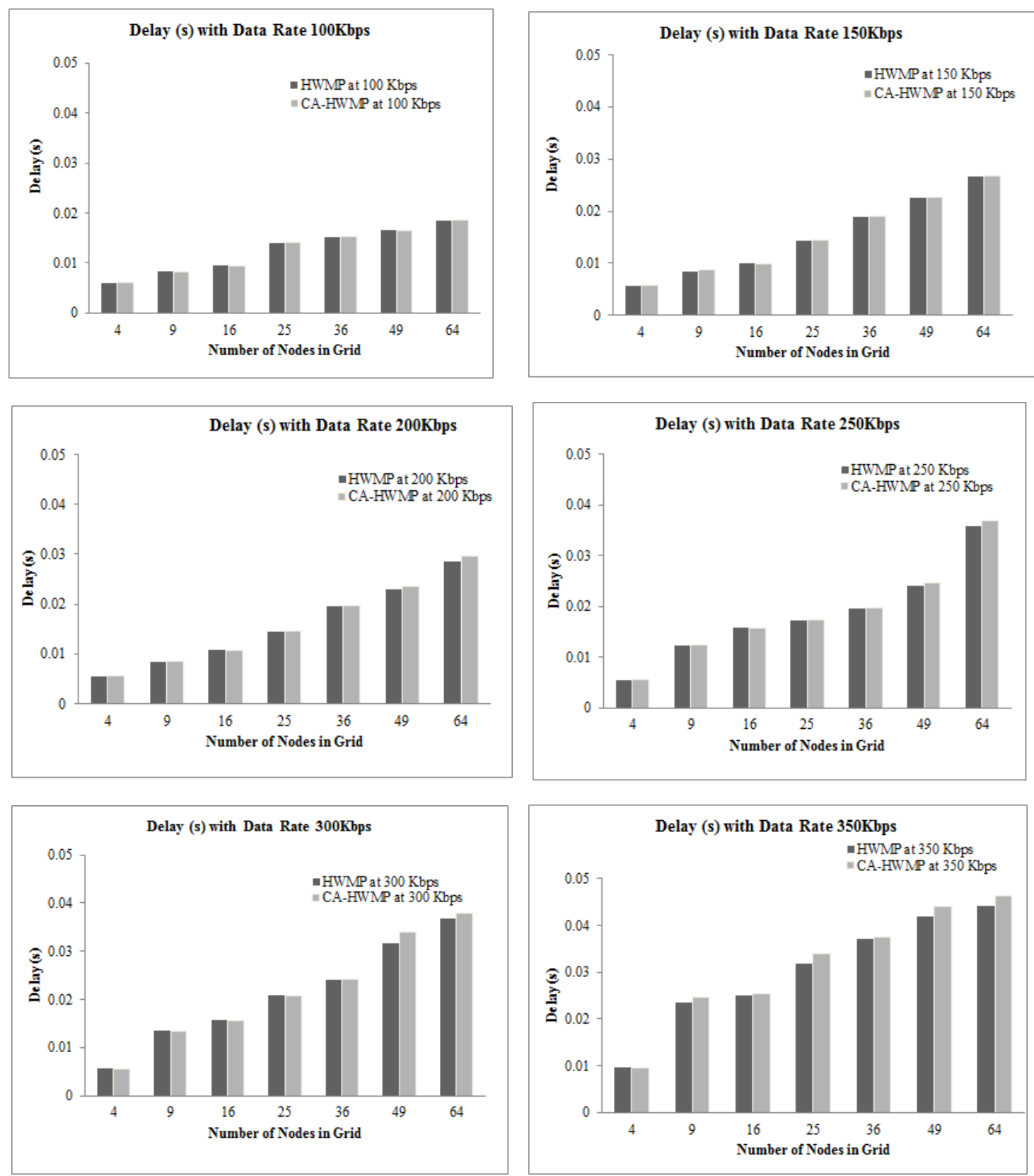

Fig. 8: (Delay Comparison of HWMP and CA-HWMP at (a) 100Kbps; (b) 150Kbps;(c) 200Kbps;(d) 250Kbps;(e) 300Kbps;(f) $350 \mathrm{Kbps}$

involved for transmission then the additional delay is added in the network.

After observing delay when we have application data-rate $150 \mathrm{Kbps}$, we increase data-rate from $150 \mathrm{Kbps}$ to $200 \mathrm{Kbps}$. By increasing the number of nodes in the network, delay increase due to increase in contention, number of hops. Figure 8 (c) shows that observed delay in both protocols is same or negligible greater delay in the case of CA-HWMP. When we have 49, and 64 nodes in the network, packet lost ratio increases due to contention, collisions, re-transmissions and increase in number of hops, therefore, delay observed in HWMP is less. CAHWMP performs better, and increase packet delivery fraction, as delay calculated on receiving data packets. Therefore greater delay observed in CA-HWMP, however this delay is negligible.

In the next scenario, the application data-rate is $250 \mathrm{Kbps}$ and with varied grid topology. In simulation results, we observed that delay is greater than all previous scenarios. With the increase of nodes in the network, the delay also increases because more nodes shares data when new nodes enters in network. These nodes also increase channel contention, number of hops to reach destination where channel contention on each relay node adds delay. Figure 8 (d) presented this discussed trend. As we observed that PDF of CA-HWMP is greater than HWMP, therefore delay is also computed on received packet. 
Here, we observed greater delay in case of CA-HWMP and less delay in case of HWMP. In case of CA-HWMP, when queue data stay longer in queue, and its level reached upto specified level, it re-routes data to avoid congestion and increase PDF minimum delay. CA-HWMP chooses second optimum path, which may add delay but this increase in delay is very small.

In this secnario, the application data-rate is $300 \mathrm{Kbps}$ and relay nodes vary from 4 to 64 . In this scenario like previous simulation scenarios, when new nodes enters in the network, the delay is also increases. In Figure 8 (e), bar lines of both protocols indicate insignificant differences in delay. The difference is visible when we have 49 and 64 mesh nodes in the network in both protocols, in case of CA-HWMP is greater than HWMP. it is computed on the destination node, where PDF is less in HWMP as compared to CA-HWMP. However, difference in delay is much smaller that can be ignored.

The Figure 8 (f) represents the delay chart for both protocols while varying number of nodes in the grid. In the given chart, bar lines of both charts show insignificant difference of the delay between two compared protocols. In case of CAHWMP, the received packets are greater than HWMP. End-toend delay computed on the received packet at the destination using the ratio of total delay and total packet received. It is computed by using the time difference of the time when it is sent and the time when it is received at destination. In CA-HWMP, we observed greater values of PDF with ignorant delay.

From Figure 8 (a) to (e), it is concluded that end-to-end delay increases by increasing relay nodes in the the network. With the increase in number of nodes into the network, the throughput of the network also increases due to more disseminate of data into the network, but if the traffic data is more than the network capacity, it introduces congestion in the network. This increase in relay nodes in the mesh grid, also increases interference, packet collisions and channel contention. The increase in the wait time on each relay station also added delay. We fixed all other parameters in both protocol scenarios, and change application data-rate and network density mode. We observed that when with the increase in application data-rate and relay nodes in the grid, the delay also increases due to wireless medium access issues. Packet remained in the queue until node gets a chance to transmit packets.However, the delay difference between both protocols is ignorant.

\section{COnClusions And Future Work}

The use of Internet with mobility support is at a rapid pace. WMN is one of the wireless technologies which offers high bandwidth and caters mobile users. It has the attractive feature of self-configuring, self-healing and self-organizing and is a suitable candidate for network provisioning in the areas where connectivity through wired media is comparatively difficult or lengthy process. Furthermore, the WMN is a good choice in many scenarios satisfy efficient, where other technologies cannot provide full support.

IEEE 802.11s is a MAC standard with the MAC enhancement in 802.11 MAC for WMN including enhancement of QoS, path selection, security, configuration, and management. It is first IEEE standard, which proposed a routing protocol at the MAC layer i.e. HWMP. It is the mandatory protocol and offers the advantages of both reactive and proactive approaches.

In wireless network, packet collision is generally because of the wireless communication issues, such as contention for channel access, delayed packet in queue due to long wait etc. In IEEE 802.11, one node can only transmit data at a time, due to shared channel characteristics. This restriction adds a significant delay with the increment of the number of hops. The increase in channel contention delay, and queue length leads to congestion. In a WMN, as traffic is aggregated at the MP, MPs near MPP have greater traffic load as compared to other nodes. Therefore, in the absence of any congestion control mechanism, nodes at the outer edges of the network undergo low throughput and increased packet loss. To solve this problem many researchers have proposed algorithms like Total Congestion Control (TCC), Link Selective Congestion Control (LSCC) and Path Selective Congestion Control (PSCC), but every technique have their own pros and cons. In TCC, on receiving CCNF, STA stop sending data to all neighboring nodes although STA can send data to other neighboring nodes. This approach wastes the available bandwidth and add delay. In PSCC, on receiving CCNF the STA only block sending data for the specific link but can receive data. In this scenario, when congestion occurs in the network, STA when it gets congested, it cannot broadcast CCNF message to blocked link. Hence packets drop due to queue overflow. The third is PSCC, which block the specific path on receiving CCNF. For the announcement of specific destination, this algorithm requires modification in the standard CCNF. On receiving modified CCNF a node only block sending data for a specific destination, but it continuously receives data for that specific client. The scenario becomes more complicated when CCNF frame is further broadcasted to immediate node in a continuous chain.

To handle congestion at the MAC layer, we proposed a congestion avoidance technique named Congestion Avoidance Hybrid Wireless Mesh Protocol (CA-HWMP). In this protocol, when node queue level reached to a specified threshold value, it broadcasts $\mathrm{CCNF}$ to its immediate neighbors before reaching to congestion state. The nodes present in its neighbor reroute all traffic on congested node from alternate path. For comparison, we have selected our proposed approach using IEEE 802.11s WMN with its mandatory routing protocol i.e. HWMP. For performance evaluation, we used NS3 which is based on object oriented language $C++$ and a scripting language Python. We evaluated our proposed protocol through PDF and average end-to-end delay. We also noticed this effect on the different node grids by gradually varying the environment from sparse to dense mode. From the comparison, it is concluded that CA-HWMP performs better than HWMP in term of greater throughput and PDF. However, CA-HWMP offers negligibly higher delay than HWMP. The increased delay is caused due to selection of alternate path, which may not be the optimal one. However, it offers almost same delay due to congestion as compared to default protocol.

During the evaluation, we found some limitations of our proposed technique and default routing protocol. Scalability is one issue with both protocols. Although CA-HWMP performs better but these both protocols perform good with limited number of mesh nodes. The HWMP did nothing with congestion, 
but the proposed technique also has limitations which include wait by blocking node in case there is no alternate path to re-route. In such cases, a blocked node waits for the CCNF expiry time to initiate transmission to an already established path which was blocked due to congestion. For the extension of this work, it is recommended to cater the congestion problem in the absence of alternate path. A hybrid technique can also aim to solve this problem. The comparison of our proposed routing protocol with existing congestion control protocol can also consider as its future work.

\section{REFERENCES}

[1] Y.-C. Du, Y.-Y. Lee, Y.-Y. Lu, C.-H. Lin, M.-J. Wu, C.-L. Chen, and T. Chen, "Development of a Telecare System Based on Zigbee Mesh Network for Monitoring Blood Pressure of Patients with Hemodialysis in Health Care Centers," Journal of medical systems, vol. 35, no. 5, pp. 877-883, 2011

[2] C. S. Wang and Y.-R. Tzeng, "A Wireless Networking Technologies Overview over Ubiquitous Service Applications," in Fourth International Conference on Networked Computing and Advanced Information Management (NCM'08), vol. 1. IEEE, 2008, pp. 156-161.

[3] V. C. Gungor and F. C. Lambert, "A Survey on Communication Networks for Electric System Automation," Computer Networks, vol. 50, no. 7, pp. 877-897, 2006.

[4] Z. Chen, L. Chen, Y. Liu, and Y. Piao, "Application Research of Wireless Mesh Network on Earthquake," in International Conference on Industrial and Information Systems (IIS'09). IEEE, 2009, pp. 1922.

[5] M. S. Akbar, M. S. Khan, K. A. Khaliq, A. Qayyum, and M. Yousaf, "Evaluation of IEEE $802.11 \mathrm{n}$ for Multimedia Application in VANET," Procedia Computer Science, vol. 32, pp. 953-958, 2014.

[6] J. Ishmael, S. Bury, D. Pezaros, and N. Race, "Deploying Rural Community Wireless Mesh Networks," IEEE Internet Computing, vol. 12, no. 4, pp. 22-29, 2008.

[7] M. Seyedzadegan, M. Othman, B. M. Ali, and S. Subramaniam, "Wireless Mesh Networks: WMN Overview, WMN Architecture," in International Conference on Communication Engineering and Networks IPCSIT, vol. 19, 2011.

[8] M. L. Sichitiu, "Wireless Mesh Networks: Opportunities and Challenges," in Proceedings of World Wireless Congress, 2005.

[9] M. S. Akbar, K. A. Khaliq, and A. Qayyum, "Vehicular MAC Protocol Data Unit (V-MPDU): IEEE 802.11 p MAC Protocol Extension to Support Bandwidth Hungry Applications," in Vehicular Ad-hoc Networks for Smart Cities. Springer, 2015, pp. 31-39.

[10] T. Sprodowski and J. Pannek, "Stability of distributed MPC in an intersection scenario," in Journal of Physics: Conference Series, vol 659, no. 1. IOP Publishing, 2015, p. 012049.

[11] S. Badombena-Wanta and E. Sheybani, "Mobile Communications for Development: Enabling Strategic and Low-cost e-applications for Rural and Remote Areas," in IEEE Wireless Telecommunications Symposium (WTS), 2010, pp. 1-7.

[12] M. Portmann and A. A. Pirzada, "Wireless Mesh Networks for Public Safety and Crisis Management Applications," IEEE Internet Computing, vol. 12 , no. 1 , pp. 18-25, 2008.

[13] W. Guo and M. Zhou, "An Emerging Technology for Improved Building Automation Control," in IEEE International Conference on Systems, Man and Cybernetics (SMC 2009). IEEE, 2009, pp. 337-342.

[14] A. Yarali, B. Ahsant, and S. Rahman, "Wireless Mesh Networking: A Key Solution for Emergency \& Rural Applications," in IEEE Second International Conference on Advances in Mesh Networks (MESH 2009), 2009, pp. 143-149.

[15] K. A. Khaliq, A. Qayyum, and J. Pannek, "Methodology for Development of Logistics Information and Safety System Using Vehicular Adhoc Networks," in Springer Dynamics in Logistics, 2017, pp. 185195.
[16] K. A. Khaliq, A. Qayyum, J. Pannek, "Synergies of Advanced Technologies and Role of VANET in Logistics and Transportation," International Journal of Advanced Computer Science and Applications(IJACSA), vol. 7, no. 11, pp. 359-369, 2016. [Online]. Available: http://dx.doi.org/10.14569/IJACSA.2016.071148

[17] X. Wang and A. O. Lim, "IEEE $802.11 \mathrm{~s}$ Wireless Mesh Networks: Framework and Challenges," Elsevier Ad Hoc Networks, vol. 6, no. 6, pp. 970-984, 2008.

[18] I. F. Akyildiz, X. Wang, and W. Wang, "Wireless Mesh Networks: A Survey," Elsevier Computer Networks, vol. 47, no. 4, pp. 445-487, 2005.

[19] A. B. Forouzan, Data Communications \& Networking (sie). Tata McGraw-Hill Education, 2007.

[20] R. Karrer, A. Sabharwal, and E. Knightly, "Enabling Large-scale Wireless Broadband: The Case for TAPs," ACM SIGCOMM Computer Communication Review, vol. 34, no. 1, pp. 27-32, 2004

[21] “IEEE 802.11s/D8.0,” Draft Standard, Tech. Rep., 2009.

[22] H. Aoki, S. Takeda, K. Yagyu, and A. Yamada, "IEEE 802.11 s Wireless LAN Mesh Network Technology," NTT DoCoMo Technical Journal, vol. 8, no. 2, pp. 13-21, 2006.

[23] K. Shi, Y. Shu, and J. Feng, "A MAC layer Congestion Control Mechanism in IEEE 802.11 WLANs," in Fourth International Conference on Communications and Networking in China (ChinaCOM 2009). IEEE, 2009, pp. 1-5.

[24] K. A. Khaliq, M. S. Akbar, A. Qayyum, E. Elahi, and A. Zaheer, "Congestion Avoidance Hybrid Wireless Mesh Protocol (CA-HWMP) for IEEE 802.11s," Elsevier Procedia Computer Science, vol. 32, pp. 229-236, 2014, The 5th International Conference on Ambient Systems, Networks and Technologies (ANT-2014). [Online]. Available: http://www.sciencedirect.com/science/article/pii/S187705091400619X

[25] K. A. Khaliq, S. Hussain, A. Qayyum, and J. Pannek, "Novel Data Link Layer Encoding Scheme for Multi-hop Wireless Mesh Network," Procedia Computer Science, vol. 52, pp. 665-669, 2015.

[26] D. Fu, B. Staehle, R. Pries, and D. Staehle, "On The Potential of IEEE $802.11 \mathrm{~s}$ Intra-mesh Congestion Control," in ACM Proceedings of the 13th ACM international conference on Modeling, analysis, and simulation of wireless and mobile systems, 2010, pp. 299-306.

[27] J. Camp and E. Knightly, "The IEEE $802.11 \mathrm{~s}$ Extended Service Set Mesh Networking Standard," IEEE Communications Magazine, vol. 46, no. 8, pp. 120-126, 2008.

[28] G. Feng, F. Long, and Y. Zhang, "Hop-by-Hop Congestion Control for Wireless Mesh Networks with Multi-channel MAC," in IEEE Global Telecommunications Conference (GLOBECOM 2009), 2009, pp. 1-5.

[29] A. Raniwala, D. Pradipta, and S. Sharma, "End-to-End Flow Fairness over IEEE 802.11-based Wireless Mesh Networks," in IEEE 26th IEEE International Conference on Computer Communications (INFOCOM 2007), 2007, pp. 2361-2365.

[30] M. Ahmed and K. A. Rahman, "Novel Techniques for Fair Rate Control in Wireless Mesh Networks," International Journal, vol. 3, 2012.

[31] S. Rangwala, A. Jindal, K.-Y. Jang, K. Psounis, and R. Govindan, "Understanding Congestion Control in Multi-hop Wireless Mesh Networks," in ACM Proceedings of the 14th ACM International Conference on Mobile Computing and Networking, 2008, pp. 291-302.

[32] B. Staehle, M. Bahr, D. Fu, and D. Staehle, "Intra-mesh Congestion Control for IEEE 802.11 s Wireless Mesh Networks," in 21st IEEE International Conference on Computer Communications and Networks (ICCCN 2012), 2012, pp. 1-7. 